\title{
Intracellular AFP Blocks All-trans retinoic acid induced ATG7 expression and Autophagy in Hepatocellular carcinoma cells
}

Shanshan Wang ( $18610294297 @ 163 . c o m$ )

Captital medical universtiy

Rilu Feng

Heidelberg University

Ying Shi

Captital Medical University

Dexi Chen

Capital Medical University

Honglei Weng

Heidelberg university

Huiguo Ding

Capital Medical University

Chenguang Zhang

Capital Medical University

Research

Keywords: HCC, ATRA, RAR, AFP, ATG7, Autophagy, Apoptosis

Posted Date: April 22nd, 2020

DOl: https://doi.org/10.21203/rs.3.rs-23652/v1

License: (c) (i) This work is licensed under a Creative Commons Attribution 4.0 International License.

Read Full License 


\section{Abstract}

Background: Retinoic acid and retinoid acid receptor (RA-RAR) signaling exhibits suppressive functions in the progression of hepatocellular carcinoma (HCC) through multiple mechanisms. However, whether RARAR signaling induces autophagy that contributes its anti-tumor activity in HCC remains elusive.

Methods: The effects of RA-RAR pathway were investigated in two HCC cell lines: AFP positive PLC/PRF/ 5 cells and AFP negative HLE cells. Cell autophagy, apoptosis and proliferation were analyzed by Western blotting, co-immunoprecipitation (CoIP), Immunofluorescence staining, chromatin immunoprecipitation (ChIP), Caspase-3 activity and Cell Counting Kit (CCK)-8.

Results: ATRA dosage-dependently induced high levels of cell autophagy through its specific nuclear receptor RAR in both the PLC/PRF/5 and HLE cells. ChIP assay showed that RAR bind to response elements of key autophagy-initiated gene autophagy-relevant protein (ATG) 7 gene in the 5 '-flanking region. Analyses based on ColP further revealed that AFP formed complex with RAR in PLC/PRF/ 5 cells. Knockdown of AFP reduced the AFP and RAR combination, and thus up-regulated the expression of ATG7 gene and cell autophagy. Interestingly, in the HLE cells, AFP overexpressed and combination with RAR resulted in down-regulated ATG7 gene expression and reduction of cell autophagy. In both cell lines, ATRA inhibited cell proliferation and induced cell apoptosis, which was impacted by AFP action.

Conclusion: The current study indicated that autophagy participated in the functionality of ATRA on HCC cells and AFP is a key regulator of ATRA induced autophagy through forming complexes with RAR in HCC cells.

\section{Background}

Alpha-fetoprotein (AFP) is the most widely used biomarker for clinical diagnosis of HCC, the fifth most common malignancy and the third leading cause of cancer related death worldwide [1]. Dynamics of serum AFP closely related to the development of the tumor [2]. Besides its clinical utilization as a biomarker, growing evidence has revealed that intracellular AFP functioned as an important signaling molecule, through interaction with a list of proteins, to promote the progression of HCC [3-5]. A series work by us showed that AFP could perturb RA-RAR signaling through interaction with RARa, resulting in transcriptional dysregulation of RAR targets, like survivin [3], Fn14 [4], GADD45 [5], GADD153 [6] etc. in HCC cells. ATRA or its chemical derivatives have long been tested as candidates for treatment of HCC as single reagent or in combination with other clinically used drugs $[7,8]$, the results was, however, far to be satisfactory, which could be partially attributed to the perturbation of AFP and the resultant dysregulation of RAR target genes. Given the profound effect of ATRA on HCC cells and the broad distribution of retinoic acid response elements (RAREs) in human genome, whether other target genes of RA-RAR signaling and related biological process is regulated by AFP in HCC cells is tempting to investigation.

Macroautophagy (referred to as autophagy hereafter) is a conserved degradation system for damaged, misfolded, or senescent cellular components, like organelles or certain proteins to maintain cellular 
homeostasis [9]. About 40 autophagy related genes (ATGs) have been identified to date and participate in the whole process of autophagy that mainly composed of initiation and elongation of the phagophore, autophagosome formation, autophagosome fusion with lysosomes and final degradation of the intracapsular products, in a highly ordered manner [10]. Important signaling molecules like AMPK, mTOR, $\mathrm{PI} 3 \mathrm{~K} / \mathrm{Akt}$ etc. showed potent regulation on autophagy [11]. For example, mTORC1 inhibited autophagosome formation elicited by ULK1 (ATG1) while activated AMPK was able to inhibit mTORC1 and directly phosphorylates ULK1, leading to autophagy initiation [12]. RA-RAR signaling has also been implicated in the modulation of autophagy through multiple mechanisms in different cell types. In acute promyelocytic leukemia (APL) cells, ATRA was able to induce autophagy through inhibition of mTOR pathway, which contributed to the degradation of the fusion oncoprotein PML/RARa, resulting in cell differentiation and the remission of the tumor $[13,14]$. In breast cancer cells, ATRA was reported to induce autophagy dependent on RARa, and ablation of autophagy promoted ATRA induced apoptosis of the cancer cells [15]. Fang et al. also suggested induction of autophagy and expression of a panel of ATGs by ATRA in Hepa1-6 mouse hepatoma cells [16], however, the generality of autophagy induction by ATRA in HCC and the underlying mechanism remains to be further addressed.

Conventional chemotherapeutic drugs for HCC like doxorubicin, oxaliplatin, cisplatin have all been reported to induce autophagy in vitro and in vivo that seemed be protective for the cells under treatment, for inhibition of autophagy was able to enhance the anti-tumor activity of these drugs [17]. Multiple ATGs and related signaling pathways were shown to regulate sensitivity of HCC cells to chemo- or targeted reagents, which might hold potential therapeutic potentials [18]. We recently provided intriguing evidence that AFP played a suppressive role in the maintenance of the basal level of autophagy in HCC cells through interaction with PTEN, which led to inhibition of the phosphatase activity of PTEN and subsequent over-activation of PI3K/Akt/mTOR, and finally promoted cell survival [19]. As AFP also interacted with RARa and perturbed RA-RAR signaling as well as the anti-tumor effect of ATRA in HCC cells, whether this perturbation also participates in regulation of cell autophagy is of great interest to be investigated.

In the present study, we found that ATRA robustly induced autophagy in human HCC cells through t RAR mediated transcriptional up-regulation of ATG7, an essential ATG for autophagosome initiation, which played a protective role for ATRA treated cells. Furthermore, AFP interacted with RARa and attenuated its regulation on ATG7 expression and autophagy. Our results was supposed to be helpful for developing novel therapeutics for HCC composed of ATRA and autophagy inhibition reagents, where the level of AFP needs to be taken into consideration.

\section{Materials And Methods}

\section{Cell lines}

AFP-producing hepatocellular carcinoma cell line PLC/PRF/5 cells and AFP-non producing cell line HLE were both maintained in a $5 \% \mathrm{CO}_{2}$ incubator and cultured in DMEM medium supplemented with $10 \%$ 
FCS.

\section{Western blotting}

For western blotting, total cell proteins from each sample were extracted with radioimmunoprecipitation (RIPA, Thermofisher, Waltham, MA, USA) cell lysis buffer containing protease inhibitor cocktail (CST, Beverly, MA, United States), $15 \mu \mathrm{g}$ of which were then subjected to $12 \%$ SDS-PAGE. Electrophoretic transfer of proteins from gels onto nitrocellulose membrane was carried out in a transblotting cell. Membranes were blocked by immersing in $5 \%$ nonfat milk (w/v) /PBS for 1 hour, and then incubated with primary antibodies at $4{ }^{\circ} \mathrm{C}$ for overnight. After rinsing with $\mathrm{PBS} / 0.1 \%$ Tween-20, membranes were incubated with horseradish peroxidase-conjugated secondary Ab. The signals were visualized by incubation with the Enhanced Chemiluninescence kit and exposure on an X-ray film. Primary and secondary antibodies used in this study are listed in Table 1. 
Table 1

Antibodies and Sequences of oligonucleotides

\section{Primary and sencondary Antibodies}

\begin{tabular}{|c|c|c|c|}
\hline \multicolumn{2}{|l|}{ P62 } & Cell Signaling Technology & CST-5114S \\
\hline \multicolumn{2}{|l|}{ LC3 } & Sigma-Aldrich & L7543-100UL \\
\hline \multicolumn{2}{|l|}{ ATG7 } & Cell Signaling Technology & CST-8558S \\
\hline \multicolumn{2}{|l|}{ RAR } & Santa Cruz Biotechnology & sc773 \\
\hline \multicolumn{2}{|l|}{ AFP } & Santa Cruz Biotechnology & sc8399 \\
\hline \multicolumn{2}{|l|}{ HDAC } & Cell Signaling Technology & CST-3949 \\
\hline \multicolumn{2}{|l|}{ GAPDH } & Cell Signaling Technology & CST-2118S \\
\hline \multicolumn{2}{|c|}{ Goat anti-rabbit IgG-HRP } & Santa Cruz Biotechnology & sc-2004 \\
\hline \multicolumn{2}{|c|}{ Goat anti-mouse IgG-HRP } & Santa Cruz Biotechnology & sc-2354 \\
\hline \multicolumn{2}{|c|}{ Goat anti-Rabbit IgG Alexa Fluor 488} & Invitrogen & R37116 \\
\hline \multicolumn{2}{|c|}{ Goat anti-Mouse IgG Alexa Fluor 594} & Invitrogen & R37121 \\
\hline \multicolumn{4}{|c|}{ Oligonucleotides sequences } \\
\hline \multicolumn{4}{|c|}{ Primers for RT-qPCR } \\
\hline \multirow[t]{2}{*}{ ATG7 } & Sense & \multicolumn{2}{|c|}{ 5'-AAGAAATAATGGCGGCAGCT-3' } \\
\hline & Antisense & \multicolumn{2}{|c|}{ 5'-ACCCAACATCCAAGGCACTA-3' } \\
\hline \multirow[t]{2}{*}{ GAPDH } & Sense & \multicolumn{2}{|c|}{ 5'-TGAAGGTCGGAGTCAACGGA-3' } \\
\hline & Antisense & \multicolumn{2}{|c|}{ 5'-CCTGGAAGATGGTGATGGGAT-3' } \\
\hline \multicolumn{4}{|c|}{ Primers for ChIP-qPCR } \\
\hline \multirow[t]{2}{*}{ ATG7 } & Sense & \multicolumn{2}{|c|}{ 5'-TTGGGTTGTTGCATTTCTGA-3' } \\
\hline & Antisense & \multicolumn{2}{|c|}{ 5'-CCATCCTAATTGGCCTGTGT-3' } \\
\hline \multicolumn{4}{|c|}{ AFP-shRNA } \\
\hline Sense & \multicolumn{3}{|c|}{$\begin{array}{l}\text { 5'- } \\
\text { CACCGAACGTGGTCAATGTATAATTCAAGAGATTATACATTGACCACGTTCTTTTTTG } \\
3^{\prime}\end{array}$} \\
\hline Antisense & \multicolumn{3}{|c|}{$\begin{array}{l}\text { 5'- } \\
\text { GATCCAAAAAAGAACGTGGTCAATGRATAATCTCTTGAATTATACATTGACCACGTTC- } \\
3^{\prime}\end{array}$} \\
\hline
\end{tabular}

Immunofluorescence staining 
Cells were seeded on coated glass coverslips, then fixed with $4 \%$ paraformaldehyde. After incubated with PBST buffer (containing $0.05 \%$ triton-100, 0.5\% BSA in PBS) at room temperature for 10 min, the cells were washed with PBS twice followed with blocking using 1\% BSA at room temperature for $10 \mathrm{~min}$. The primary antibodies were then added and incubated at $4{ }^{\circ} \mathrm{C}$ overnight. After rinsing with PBST for 10 min, secondary antibodies, Alexa Fluor 594 and 488 (Thermo Fisher Scientific) were incubated with cells for one hour in room temperature followed by addition of DAPI for counterstaining of the nuclei. Cells images were captured with a Laser Confocal Microscope (Leica TCS STED-3X).

\section{Quantitative real-time reverse transcription PCR (RT-qPCR)}

Expression of ATG7 at the mRNA level was evaluated by quantitative real-time reverse transcription PCR (RT-qPCR) assay. Briefly, total RNA was extracted from HCC cells using the RNeasy Mini Kit (Qiagen, Hilden, Germany) according to the manufacturer's instructions. cDNA was then synthesized by reverse transcription of the extracted RNA using a SuperScript II First-stand Synthesis System (Invitrogen,

Carlsbad, CA). SYBR Green was used to detect the dsDNA products during the real-time PCR reaction. The mRNA content was normalized to the housekeeping gene GAPDH and fold change was calculated with the $2^{-\triangle \triangle C T}$ method. All primer sequences for RT-qPCR are listed in Table 1.

\section{Co-immunoprecipitation (Co-IP)}

Co-IP experiments for evaluation of the interaction between AFP and RAR were performed in PLC/PRF/5 and HLE cells. HCC cells were lysed in RAPI buffer containing $1 \%$ protease inhibitor cocktails. The lysates $(1 \mathrm{mg} / 500 \mu \mathrm{l})$ were then incubated with $1 \mu \mathrm{g}$ anti-AFP, anti-IgG and anti-RAR antibodys for overnight at $4^{\circ} \mathrm{C}$, then added $100 \mu \mathrm{l}$ Protein A SepHarose agarose beads (CL-4B) (17-0780-01, General Electric Company) for further incubation for at least 8 hours at $4^{\circ} \mathrm{C}$. Collected these beads and washed 3 times with $1 \mathrm{ml}$ PBS contain 1\% protease inhibitor cocktails before boiling them in the loading buffer, and then Western blotting analyses were performed .

\section{Transient transfection}

The AFP-expressing plasmid (pcDNA3.1-afp) was used to overexpress AFP in HLE cells, and the AFP interference siRNA (AFP-siRNA923) was employed to knockdown endogenous AFP in PLC/PRF/5 cells. ATG7 siRNA (sc-41447) and Con siRNA (sc-37707) were used to silece the ATG7 mRNA in the PLC/RCF/5 and HLE cells. In brief, HLE were transfected with pcDNA3.1-afp or control vector, and PLC/PRF/5 cells were transfected with AFP-shRNA923 or scramble control shRNA with Lipofectamine 3000 (Invitrogen, Carlsbad, CA) according to the manufacturer's instructions. After $24 \mathrm{~h}$ of transfection, the expression of AFP and ATG7 were evaluated by Western blotting and the interaction of AFP and RAR was examined by Co-IP. PLC/RCF/5 and HLE cells were transfected with ATG7 siRNA or Control siRNA for $24 \mathrm{~h}$, followed with ATRA treatment for another 24 hours, and then subjecte to functional evaluation.

\section{Chromatin immunoprecipitation and PCR (ChIP-PCR)}

ChIP was performed to verify the capacity of RAR for binding to the 5'regulatory region of ATG7 gene. Briefly, HCC cells were cross-linked in $1 \%$ formaldehyde/PBS for 10 min at $37^{\circ} \mathrm{Cand}$ were then washed 
twice with ice-cold PBS prior to be lysed. Chromatin fragments ranging from 200 to $1000 \mathrm{bp}$ were obtained by sonication (SCIENTZ, China). The solution containing chromatin fragments was then incubated overnight at $4{ }^{\circ} \mathrm{C}$ with anti-RAR or anti-lgG. The chromatin-antibody complexes were then washed, eluted and reverse cross-linked at $65^{\circ} \mathrm{C}$ for $6 \mathrm{~h}$. The eluted DNA was purified with the phenolchloroform. Immunoprecipitated chromatin was analyzed with PCR using primers targeting the human ATG7 promoter containing RAR binding motif. The primers used for ChIP-PCR are listed in Table 1.

\section{Evaluation of fluorescent LC3 puncta}

The tandem mRFP-GFP-LC3 adenoviruses construct was obtained from Hanbio Inc (Shanghai, China) and was used to evaluate autophagy induction. Briefly, $5 \times 10^{4} \mathrm{PLC} / \mathrm{PRF} / 5$ or HLE cells cultured on coverslips in 24- well microplates were transduced with mRFP-GFP-LC3 adenoviruses at $50 \mathrm{MOI}$. Two hours after transduction, removed the adenovirus by washing the cells with PBS, and then maintained the

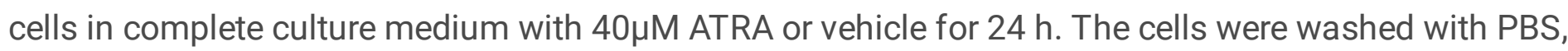
fixed with $4 \%$ paraformaldehyde, and viewed under a fluorescence microscope. The number of mRFP, GFP and GFP-mRFP (yellow) dots were determined by manual counting of fluorescent puncta in five fields from three different cells. The number of dots per cell was obtained by dividing the total number of dots by the number of cell in each microscopic field.

\section{Determination of viability}

Cell Counting Kit (CCK)-8 (CK04, Dojindo) was used to assess the cells viability. PLC/PRF/5 and HLE cells were seeded into 96-well microplates, and transfected with Si-Con or Si-ATG7. $24 \mathrm{~h}$ after transfection, the cells were subjected to $40 \mu \mathrm{M}$ ATRA and solvent for another 24 hours. The absorbance at $570 \mathrm{~nm}$ wavelength was measured with a Universal Microplate Reader (EL x800). The percentage of cell viability was calculated as (A570 sample-background)/ (A570 control-backgroud)

\section{Caspase- 3 activation assay}

PLC/PRF/5 and HLE cells were homogenized in lysis buffer. Thereafter, $30 \mu \mathrm{L}$ lysates were added to a white 96-well plate, and then mixed with $60 \mu \mathrm{L}$ assay buffer. $90 \mu \mathrm{L}$ assay buffer was added into the blank well. After incubation for 10 minutes at $37^{\circ} \mathrm{C}$, each well was added $10 \mu \mathrm{L}$ AC-DEVD-AFC at final concentration of $10 \mu \mathrm{g} / \mathrm{mL}$, followed with further incubation for $1 \mathrm{~h}$ at $37^{\circ} \mathrm{C}$ in the dark. The luminescence was measured using a Imaging Multi-Mode Reader (BioTek). The changes of caspase-3 activity was calculated as [(A400 sample-blank $) /$ Protein concentration $] /[(\mathrm{A} 400$ control-blank $) /$ Protein concentration].

\section{Statistical Analysis}

The results of at least three separate experiments are presented as the mean \pm s.d. Statistical significance was determined using the one-way and two-way ANOVA tests (SPSS 16 software).

\section{Results}




\section{ATRA induced autophagy in PLC/PRF/5 and HLE cells}

To investigate if ATRA was able to induce autophagy in HCC cells, western blotting, and immunofluorescence assays were used to detect the molecular markers of autophagy and morphological alterations in PLC/PRF/5 and HLE cells. HCC cells were treated with ATRA at $40 \mu \mathrm{M}$ for $24 \mathrm{~h}$. Ethyl Alcohol (Alc), the solvent of ATRA, was used as negative control. As shown in Fig. 1A, ATRA treatment significantly promoted cell autophagy, in both PLC/PRF/5 and HLE cells, as demonstrated by upregulation of LC3-II and decrement of P62/SQSTM1 at the protein level. To further evaluate the autophagic flux in ATRA treated HCC cells, we employed the mRFP-GFP-LC3 adenovirus vectors. PLC/PRF/5 and HLE cells transfected with mRFP-GFP-LC3 adenovirus were added with $40 \mu \mathrm{M}$ ATRA and cultured for $24 \mathrm{~h}$. As shown in Fig. 1B, numbers of GFP and mRFP dots per cell were both significantly increased under ATRA treatment, suggesting that ATRA could increases autophagic flux. Immunofluorescence analyses further confirmed that ATRA significantly reduced the expression of P62/SQSTM1 in both PLC/PRF/5 and HLE cells (Fig. 1C). All these results indicated that ATRA induced autophagy in HCC cells. The activation of RA-RAR signaling in HCC cells was further verified with nuclear accumulation of RAR as demonstrated with western blot analyses for nuclear proteins (supplementary Fig. 1A and 1B) and cellular immunofluorescence (supplementary Fig. 1C and 1D).

\section{The ATRA-RAR signaling regulated transcription of ATG7}

To further reveal the potential molecular mechanism underlying ATRA induced autophagy in HCC cells. Expressions of ATG5, Beclin and ATG7 were evaluated with RT-qPCR in PLC/PRF/5 and HepG2 cells. According to preliminary experimental results (supplementary Fig. 2), expression of ATG7, an E1-like activating enzyme that are indispensable for autophagosome formation [20], was most significantly upregulated under ATRA treatment in both HCC cell lines. We thus focused on potential regulation of ATG7 by ATRA-RAR in the following studies. Western blotting showed that ATRA induced robust increment of ATG7 in both PLC/PRF/5 (Fig. 2A) and HLE (Fig. 2A') cells, in a dose-dependent, reaching maximum at $40 \mu \mathrm{M}$. Similar results were observed at the mRNA level with the qRT-qPCR assay (Fig. 2B and 2B'). Ethyl Alcohol, solvent for ATRA, did not influence the expression of the ATG7 gene (Fig. 2A, B, 2A' and 2B'). The alteration of ATG7 at the mRNA level prompted us to investigate if ATG7 was transcriptionally regulated by RAR. Two adjacent binding sequence for RAR was discovered at the proximal promoter of ATG7 (Fig. 2C). To validate RAR was able to binding to the regeion, ChIP assays were performed. As shown in Fig. 2D and 2D', RAR was able to bind to the $5^{\prime}$-flanking regions containing its responsive elements at the ATG7 promoter in both PLC/PRF/5 and HLE cells, indicating a direct transcriptional regulation of ATRARAR signaling on ATG7 via RAR.

\section{ATG7 played a protective role in HCC during ATRA treatment.}

We next investigated whether the induction of ATG7 expression and autophagy were functional in ATRA treated HCC, or merely indicators for the activity of ATRA. CCK-8 and caspase-3 activity assays were carried out. The CCK-8 results shown that the cell viability was further decreased with knockdown of 
ATG7 in response to ATRA in both PLC/PRF/5 and HLE cells (Fig. 3A and 3A'), which was accompanied with increment of caspase-3 activity (Fig. 3B and B'). These results indicated that the induced ATG7, and quite probable the autophagy, played a protective role in ATRA induced apoptosis in HCC cells.

\section{AFP interacted with RAR in hepatoma cells}

To investigate whether AFP could possibly regulate ATRA-RAR mediated autophagy, western blotting analyses were first employed to detect the endogenous expression of AFP in PLC/PRF/5 and HLE cells. As previously reported, AFP protein was undetectable in HLE cells, but robustly expressed in PLC/PRF/5 cells (Fig. 4A). Further analyses with confocal microscopy showed that AFP and RAR co-localized in cytoplasm in PLC/PRF/5 cell (Fig. 4B), but not in HLE cells (Fig. 4C), which were further confirmed by CoIP analysis (Fig. 4D and 4E).

\section{AFP perturbed ATRA-RAR signal and reduced ATG7 expression in hepatoma cells.}

To further investigate if AFP was involved in ATRA-RAR signal transduction as well as ATG7 transcription by interacting with RAR, expression of AFP was first knockdown by specific siRNA in PLC/PRF/5 cell. Immunofluorescence and confocal microscopy assays showed that AFP expression was obviously depleted upon specific siRNA transfection compared with scramble siRNA (Fig. 5A). Following AFP depletion, binding of AFP with RAR was significantly decreased as demonstrated by Co-IP assay in PLC/PRF/5 cells (Fig. 5B). On the contrary, when AFP was introduced into HLE cells with pcDNA3.1-afp vectors (Fig. 5A'), notable interaction between AFP and RAR was observed as shown by Co-IP results (Fig. 5B'), accompanied with co-localization of AFP and RAR in the cytoplasm (Fig. 5A'). One intriguing phenomena was observed that alteration of the alteration intracellular AFP level not only changed its interaction with RAR, but also exhibit a negative regulation on the protein level of RAR itself (Fig. 5A and $\left.5 A^{\prime}\right)$, which needs further investigation.

As we previously demonstrated that interaction between AFP and RAR was able to disrupt the transcriptional regulation of RAR on its targets, we wonder whether it was also the case in ATG7. Not surprisingly, when AFP was down regulated by siRNA in PLC/PRF/5 cells, the ATG7 protein level was remarkably increased as compared with the control (Fig. 5C). On the other hand, AFP expression in HLE cells showed an apparent reduction of ATG7 protein (Fig. 5C').

\section{Discussion}

In the present study, ATRA treatment robustly induced autophagy in HCC cells through transcriptional upregulation of ATG7. Mechanistically, ATRA induced nuclear accumulation of RAR, which bound onto the promoter region of ATG7 that harbors RAR binding motifs. Intracellular AFP interacted with RAR and exhibited an inhibitory effect on nuclear accumulation of RAR, resulting in down-regulation of ATG7 of HCC cells. Functional studies indicated a protective role of the induced expression of ATG7, as knockdown of ATG7 further aggravated ATRA induced cell apoptosis. 
ATRA has long been used clinically to induce differentiation of APL cells, where the relationship between ATRA and autophagy were mostly studied. An array of ATGs and important regulators of autophagy, including ATG1, ATG5, Beclin, mTOR, PI3KC3, WIPI and TFEB, DRAM etc., were implicated in ATRA induced autophagy $[14,21-24]$. In other cell types, including several other solid tumor types, ATRA was also able to induce autophagy $[15,25]$. In these studies, expression alterations of certain ATGs or signaling molecules were always displayed as the underlying mechanisms, which seemingly was not powerful enough to establish direct links between ATRA and autophagy, as the involvement and the function of RAR always lacked. For example, ATRA induced autophagy in human B cells through mTOR inhibition [26], and induced autophagy in APL cells via potent up-regulation of TFEB [23], how the inhibition or promotion occurred, directly through RAR or by other alternative pathways?? The present study directly linked ATRA and autophagy in HCC cells with RAR mediated transcriptional activation of ATG7. Of course, as ATRA was able to elicit a number of other downstream signaling pathways [27], it still cannot rule out the possibility that other regulators were also involved in ATRA induced autophagy in HCC cells.

Blockade of autophagy by knockdown Atg1, Atg5 and PI3KC3 etc. or by specific autophagy inhibitors like 3-methyladenine (3-MA) impaired ATRA induced differentiation of APL cells [14], suggesting the necessity of autophagy for the primary function of ATRA. In breast cancer cells, autophagy was reported to be cell protective and inhibition of autophagy genetically or pharmacologically resulted in robust apoptosis [15]. In the current study, as in most cases of chemotherapeutic drug treatment, ATRA induced autophagy also played a protective role for the cancer cells though overwhelmed by the potency of ATRA. Thus autophagy constituted a resistance mechanism for HCC cells to the stimuli. ATRA has been shown to induce differentiation of tumor initiating cells in HCC, and potentiated the cytotoxicity of chemotherapeutic drugs like cisplatin [28]. It has also been shown to enhance the anti-tumor activity of sorafenib through activation of AMPK [29], a potent regulator for autophagy induction. Together with the reports that most reagents for HCC treatment induced autophagy, and autophagy was cell protective in most cases, it is plausible to consider combinational use of chemotherapeutic drugs with ATRA and autophagy inhibitors like chloroquine to improve the efficacy of chemotherapy for HCC. Further in vitro and in vivo experiments are needed to address these issues.

We recently reported that AFP was able to block basal level of autophagy in HCC cells through direct sequestration of PTEN, which leads to overactivation of PI3K-Akt-mTOR cascade [19]. Together with current results, AFP was thus able to disrupt both the basal and ATRA induced autophagy through interaction of different partners (PTEN or RAR) and modulation of different autophagy regulators (PI3K/Akt/mTOR or ATG7). However, whether autophagy and AFP played identical roles in these conditions still needs further illustration. Under basal conditions, increased level of autophagy with AFP knockdown was accompanied with PTEN overactivation and increased cell apoptosis [19]. However, to what extent did autophagy contribute to increased apoptosis could not be figured out with the evidence provided; In ATRA treated conditions, ATG7 played cell protective roles and AFP blocked ATG expression. From this point of view, AFP seemed to contribute to ATRA induced cell death, which was obviously against its well-known tumor promoting function. The discrepancy could be explained that PTEN and 
RAR were both able to elicit multiple important downstream signalings or effectors to exert their potent anti-tumor functions. AFP, as an important oncoprotein for HCC, interacted with both molecules to counteract their biological functions, with autophagy induction being one of them. Interestingly, ATRA has been shown to induce expression of PTEN, which contributed its anticancer activity in APL cells [30]. Whether similar crosstalk exists, and how overexpression of AFP could orchestrate those signaling pathways in HCC required further investigation.

\section{Conclusion:}

In this study, we found that RA-RAR pathway upregulated HCC cells autophagy levels by directly regulated ATG7 gene express, and this function was inhibited by AFP, which could block RA-RAR pathway by combining with RAR. And further RA-RAR pathway influence HCC cells apoptosis and proliferation, which was impacted by AFP action too.

Taken together, all the results indicated that autophagy participated in the functionality of ATRA on HCC cells and AFP is a key regulator of ATRA induced autophagy through forming complexes with RAR in HCC cells.

\section{Abbreviations}

AFP

Alpha-fetoprotein;

ATRA

All-trans retinoic acid

RAR

Retinoic acid receptor

ATG

Autophagy related gene

HCC

Hepatocullar carcinoma

ColP

Co-immunoprecipitation

ChIP

Chromatin immunoprecipitation

CCK-8

Cell Counting Kit

RIPA

Radioimmunoprecipitation

\section{Declarations}




\section{Ethics approval and consent to participate}

Not applicable

\section{Consent for publication}

All authors read and approved the final manuscript.

\section{Availability of data and materials}

Not applicable

\section{Competing interests}

All the authors declares that they have no conflict of interest.

\section{Funding}

This study was funded by the National Natural Science Foundation of China (No.81870424; 81672725 and 81970525), and the Beijing Natural Science Foundation Program and Scientific Research Key Program of Beijing Municipal Commission of Education (KZ201810025037)

\section{Authors' contributions}

All authors contributed to the study conception and design. WS and ZC designed this study. Material preparation, data collection and analysis were performed by WS, FR, SY and CD. The first draft of the manuscript was written by $\mathrm{WS}, \mathrm{WH}$ and $\mathrm{DH}$. ZC reviewed the manuscript.

\section{Acknowledgements}

W.S. was supported by funds from the Chinese Nature Science Foundation (81870424). DH was supported by the Chinese Nature Science Foundation (81672725 and 81970525) and Beijing Nature Science Foundation Program and Scientific Research Key Program of Beijing Municipal Commission of Education (KZ201810025037). The funding bodies did not influence the content of this article.

\section{Authors' information}

Shanshan Wang: 18610294297@163.com

Rilu Feng: Rilu.Feng@medma.uni-heidelberg.de.

Ying Shi: quartz_shi@163.com

Dexi Chen: dexi09@yahoo.com

Honglei Weng: honglei.weng@medma.uni-heidelberg.de. 
Huiguo Ding: dinghuiguo@medmail.com.cn

Chenguang Zhang: chzhang@ccmu.edu.cn

\section{References}

1. Heimbach JK, Kulik LM, Finn RS, Sirlin CB, Abecassis MM, Roberts LR, Zhu AX, Murad MH, Marrero JA. AASLD guidelines for the treatment of hepatocellular carcinoma. HEPATOLOGY. 2018;67:358.

2. Wong RJ, Ahmed A, Gish RG. Elevated alpha-fetoprotein: differential diagnosis - hepatocellular carcinoma and other disorders. CLIN LIVER DIS. 2015;19:309.

3. Li M, Li H, Li C, Guo L, Liu H, Zhou S, Liu X, Chen Z, Shi S, Wei J, McNutt MA, Li G. Cytoplasmic alphafetoprotein functions as a co-repressor in RA-RAR signaling to promote the growth of human hepatoma Bel 7402 cells. CANCER LETT. 2009;285:190.

4. Wang S, Jiang W, Chen X, Zhang C, Li H, Hou W, Liu Z, McNutt MA, Lu F, Li G. Alpha-fetoprotein acts as a novel signal molecule and mediates transcription of Fn14 in human hepatocellular carcinoma. $J$ HEPATOL. 2012;57:322.

5. Zhang C, Chen X, Liu H, Li H, Jiang W, Hou W, McNutt MA, Lu F, Li G. Alpha fetoprotein mediates HBx induced carcinogenesis in the hepatocyte cytoplasm. INT J CANCER. 2015;137:1818.

6. Li C, Wang S, Jiang W, Li H, Liu Z, Zhang C, McNutt MA, Li G. Impact of intracellular alpha fetoprotein on retinoic acid receptors-mediated expression of GADD153 in human hepatoma cell lines. INT J CANCER. 2012;130:754.

7. Okuno M, Kojima S, Matsushima-Nishiwaki R, Tsurumi H, Muto Y, Friedman SL, Moriwaki H. Retinoids in cancer chemoprevention. Curr Cancer Drug Targets. 2004;4:285.

8. Shi J, Sun J, Liu C, Chai Z, Wang N, Zhang H, Cheng S. All-trans-retinoic acid (ATRA) plus oxaliplatin plus 5-fluorouracil/leucovorin (FOLFOX) versus FOLFOX alone as palliative chemotherapy in patients with advanced hepatocellular carcinoma and extrahepatic metastasis: study protocol for a randomized controlled trial. TRIALS. 2019;20:245.

9. Levy J, Towers CG, Thorburn A. Targeting autophagy in cancer. NAT REV CANCER. 2017;17:528.

10. Levine B, Kroemer G. Biological Functions of Autophagy Genes: A Disease Perspective. CELL. 2019;176:11.

11. Yang S, Yang L, Li X, Li B, Li Y, Zhang X, Ma Y, Peng X, Jin H, Li H. New insights into autophagy in hepatocellular carcinoma: mechanisms and therapeutic strategies. AM J CANCER RES. 2019;9:1329.

12. Kim J, Kundu M, Viollet B, Guan KL. AMPK and mTOR regulate autophagy through direct phosphorylation of Ulk1. NAT CELL BIOL. 2011;13:132.

13. Isakson P, Bjoras M, Boe SO, Simonsen A. Autophagy contributes to therapy-induced degradation of the PML/RARA oncoprotein. BLOOD. 2010;116:2324.

14. Wang Z, Cao L, Kang R, Yang M, Liu L, Zhao Y, Yu Y, Xie M, Yin X, Livesey KM, Tang D. Autophagy regulates myeloid cell differentiation by p62/SQSTM1-mediated degradation of PML-RARalpha 
oncoprotein. AUTOPHAGY. 2011;7:401.

15. Brigger D, Schlafli AM, Garattini E, Tschan MP. Activation of RARalpha induces autophagy in SKBR3 breast cancer cells and depletion of key autophagy genes enhances ATRA toxicity. CELL DEATH DIS. 2015;6:e1861.

16. Fang SY, Cui JJ, Gong MJ, He Y, Zhang JF, Bi Y. [Changes in autophagy during maturation and differentiation of Hepa1-6 cells induced by all-trans retinoic acid]. Nan Fang Yi Ke Da Xue Xue Bao. 2018;38:527.

17. Sheng J, Qin H, Zhang K, Li B, Zhang X. Targeting autophagy in chemotherapy-resistant of hepatocellular carcinoma. AM J CANCER RES. 2018;8:354.

18. Niu L, Liu L, Yang S, Ren J, Lai P, Chen GG. New insights into sorafenib resistance in hepatocellular carcinoma: Responsible mechanisms and promising strategies. Biochim Biophys Acta Rev Cancer. 2017;1868:564.

19. Wang S, Zhu M, Wang Q, Hou Y, Li L, Weng H, Zhao Y, Chen D, Ding H, Guo J, Li M. Alpha-fetoprotein inhibits autophagy to promote malignant behaviour in hepatocellular carcinoma cells by activating PI3K/AKT/mTOR signalling. CELL DEATH DIS. 2018;9:1027.

20. Shimizu S. Biological Roles of Alternative Autophagy. MOL CELLS. 2018;41:50.

21. Humbert M, Mueller C, Fey MF, Tschan MP. Inhibition of damage-regulated autophagy modulator-1 (DRAM-1) impairs neutrophil differentiation of NB4 APL cells. Leuk Res. 2012;36:1552.

22. Brigger D, Proikas-Cezanne T, Tschan MP. WIPI-dependent autophagy during neutrophil differentiation of NB4 acute promyelocytic leukemia cells. CELL DEATH DIS. 2014;5:e1315.

23. Orfali N, O'Donovan TR, Cahill MR, Benjamin D, Nanus DM, McKenna SL, Gudas LJ, Mongan NP. Alltrans retinoic acid (ATRA)-induced TFEB expression is required for myeloid differentiation in acute promyelocytic leukemia (APL). EUR J HAEMATOL. 2020;104:236.

24. Trocoli A, Mathieu J, Priault M, Reiffers J, Souquere S, Pierron G, Besancon F, Djavaheri-Mergny M. ATRA-induced upregulation of Beclin 1 prolongs the life span of differentiated acute promyelocytic leukemia cells. AUTOPHAGY. 2011;7:1108.

25. Patties I, Kortmann RD, Menzel F, Glasow A. Enhanced inhibition of clonogenic survival of human medulloblastoma cells by multimodal treatment with ionizing irradiation, epigenetic modifiers, and differentiation-inducing drugs. J Exp Clin Cancer Res. 2016;35:94.

26. Eriksen AB, Torgersen ML, Holm KL, Abrahamsen G, Spurkland A, Moskaug JO, Simonsen A, Blomhoff HK. Retinoic acid-induced IgG production in TLR-activated human primary $B$ cells involves ULK1-mediated autophagy. AUTOPHAGY. 2015;11:460.

27. Costantini L, Molinari R, Farinon B, Merendino N. Retinoic Acids in the Treatment of Most Lethal Solid Cancers. J CLIN MED 2020;9.

28. Zhang Y, Guan DX, Shi J, Gao H, Li JJ, Zhao JS, Qiu L, Liu J, Li N, Guo WX, Xue J, Zhou FG, Wu MC, Wang HY, Xie D, Cheng SQ. All-trans retinoic acid potentiates the chemotherapeutic effect of cisplatin by inducing differentiation of tumor initiating cells in liver cancer. J HEPATOL. 2013;59:1255. 
29. Ishijima N, Kanki K, Shimizu H, Shiota G. Activation of AMP-activated protein kinase by retinoic acid sensitizes hepatocellular carcinoma cells to apoptosis induced by sorafenib. CANCER SCI. 2015;106:567.

30. Lee YR, Yu HN, Noh EM, Kim JS, Song EK, Han MK, Kim BS, Lee SH, Park J. Peroxisome proliferatoractivated receptor gamma and retinoic acid receptor synergistically up-regulate the tumor suppressor PTEN in human promyeloid leukemia cells. INT J HEMATOL. 2007;85:231.

\section{Supplementary Figure Legends}

\section{Supplementary Fig. 1. Effect of ATRA on localization of RAR in PLC/PRF/5 and HLE cells}

Cells were treated with $40 \mu \mathrm{M}$ ATRA for $4 \mathrm{~h}$ and expression of total RAR and nuclear RAR were evaluated with Western blotting in PLC/PRF/5 (A) and HLE (B) cells. Expression and localization of RAR in PLC/PRF/5 (C) and HLE (D) cells following ATRA treatment were viewed and captured under laser confocal microscope. Nuclei and RAR were stained with DAPI (blue) and FITC (green), respectively. The image is representative of three independent experiments.

Supplementary Fig. 2. Expression alteration of certain ATGs in hepatoma cells. The expression at the mRNA level of ATGA7 (A), Beclin (B) and ATG5 (C) in PLC/PRF/5 and HepG2 cells upon ATRA treatment were determined with RT-qPCR.

\section{Figures}


A

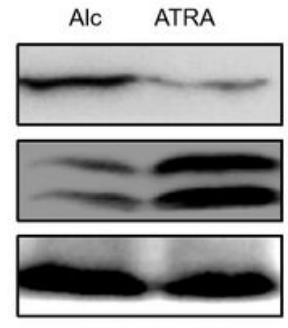

PLC/RCF/5

B

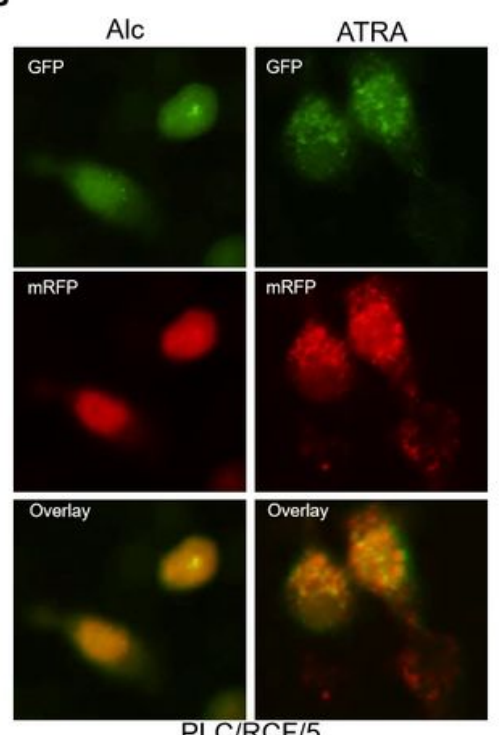

PLC/RCF/5

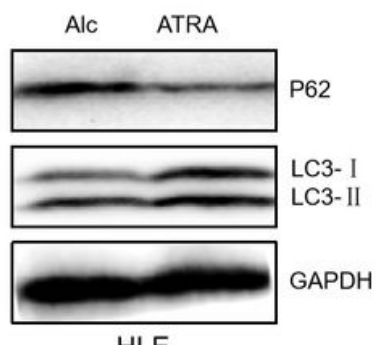

HLE

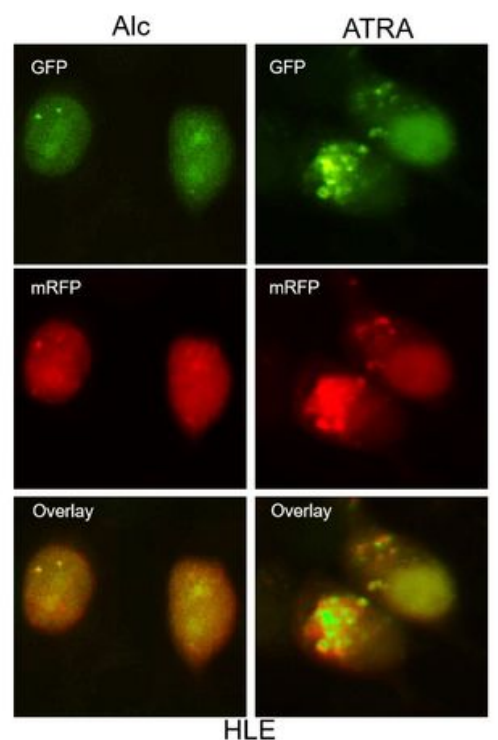

c
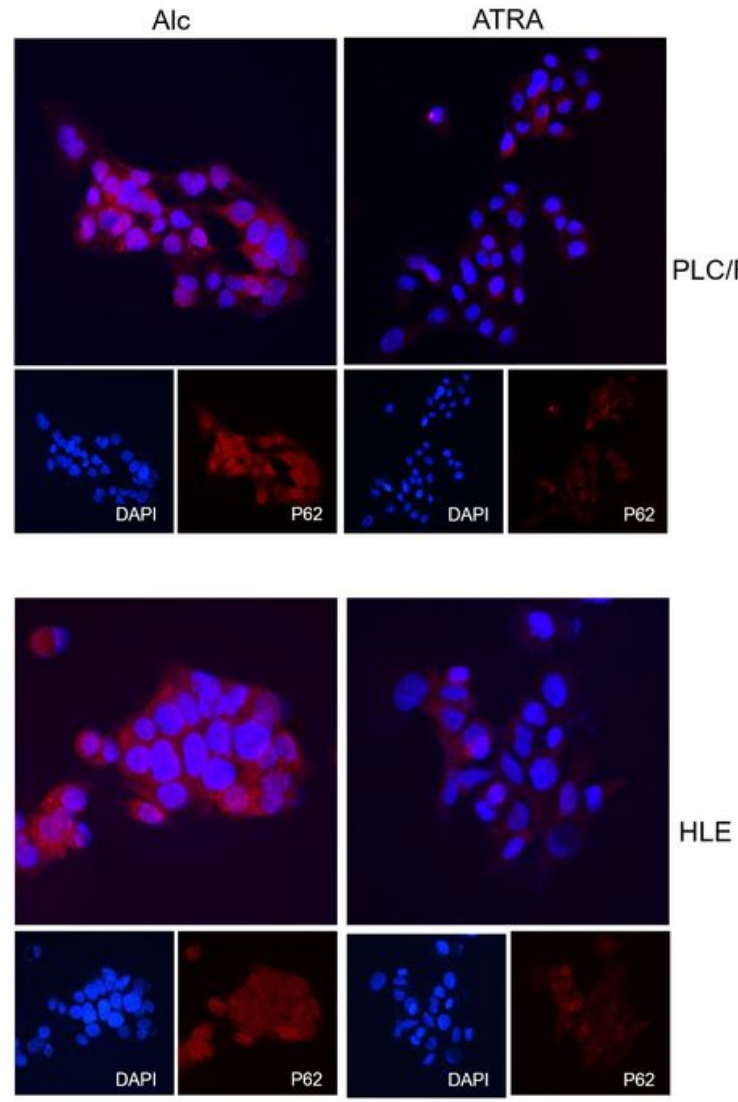

\section{Figure 1}

ATRA promoted autophagy in PLC/PRF/5 and HLE cells. A. Western blotting analyses of the expression of P62/SQSTM1 and LC3 in PLC/PRF/5 and HLE cells upon ATRA treatment for 24h. B. PLC/PRF/5 and HLE cells were transduced with tandem mRFP-GFP-LC3 adenovirus and were then subjected to ATRA for 24 h. Representative images of fluorescent LC3 puncta are shown. C. The expression of P62/SQSTM1 was detected with immunofluorescence and was observed under the fluorescence microscopy. Nuclei were stained with DAPI (blue). P62/SQSTM1 were labeled with TRITC (red). All the shown images are representative of three independent experiments. Ethyl Alcohol (Alc) was used as a solvent and as a control. 


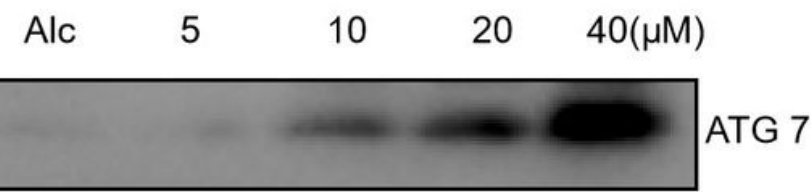

A' Alc $5 \quad 10 \quad 20 \quad 40(\mu \mathrm{M})$
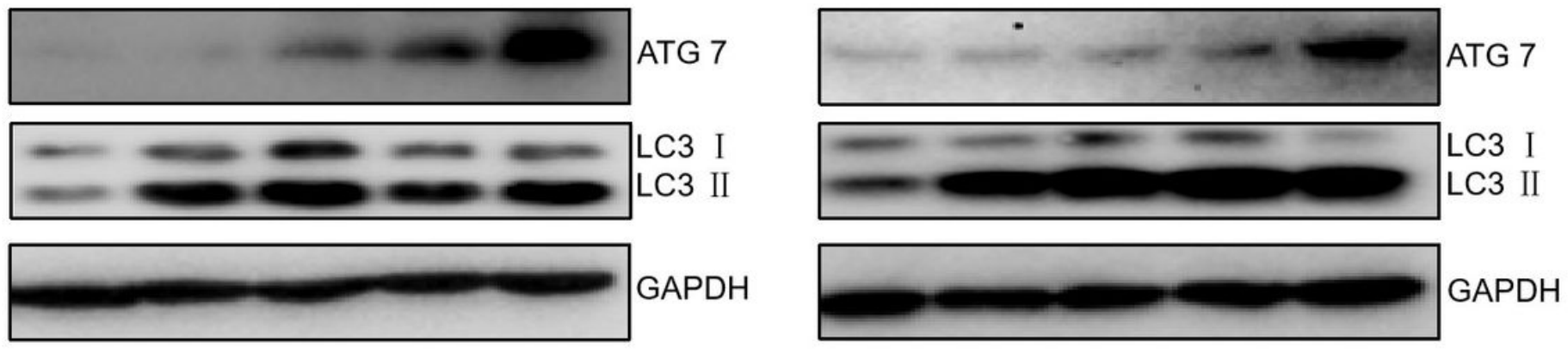

B

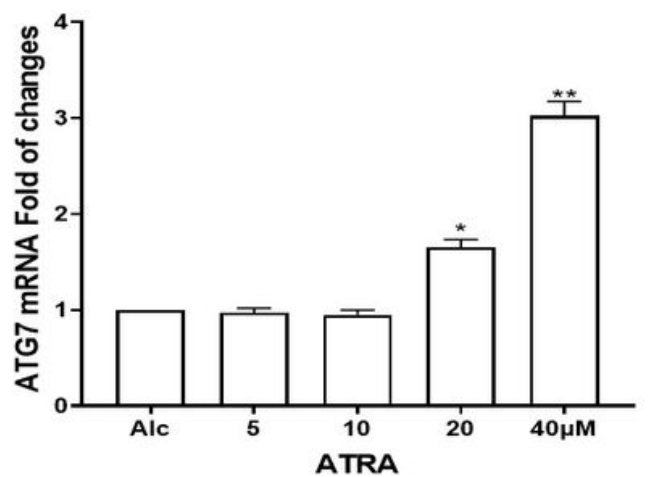

B'

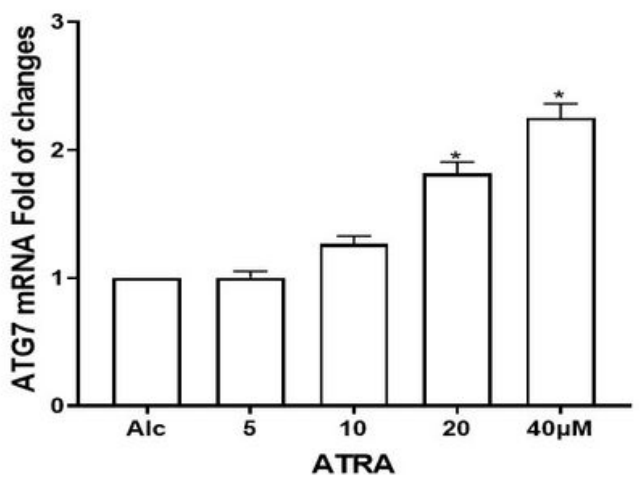

C

ATG7 gene promoter region

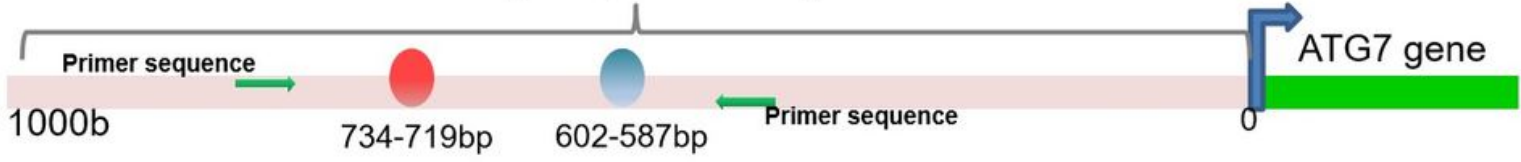

RAR binding motif on the + strand

RAR binding motif on the - strand

D

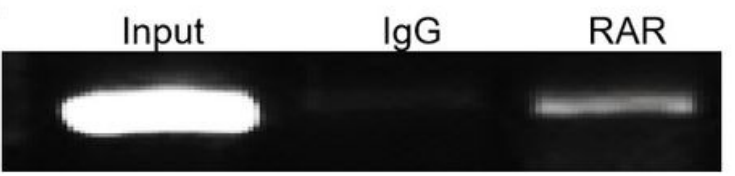

$\mathrm{PLC} / \mathrm{RCF} / 5$

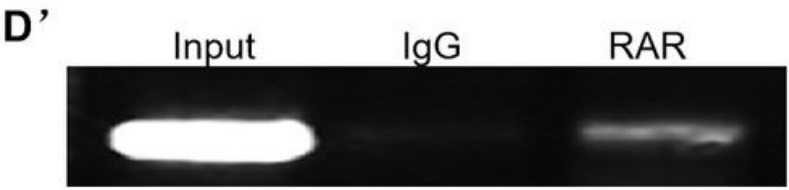

HLE

Figure 3

RAR signal cascade regulated transcription of the ATG7 in HCC cells. Various concentrations of ATRA were used to treat HCC cells for 24h. Expression of the ATG7 protein in PLC/PRF/5 (A) and HLE (A') cells was detected using Western blotting. The mRNA expression level of ATG7 gene in PLC/PRF/5 (B) and HLE (B') cells were analyzed with qRT-qPCR. (C) Schematic overview of the RAR binding sites on ATG7 promoter predicted with JASPAR website (http://jaspar.genereg.net/). ChIP assays for RAR binding onto ATG7 promoter were carried out in PLC/PRF/5 (D) and HLE (D') cells. * and \#P<0.05 and ** and \#\#P< 0.01 as compared with the control. 
A

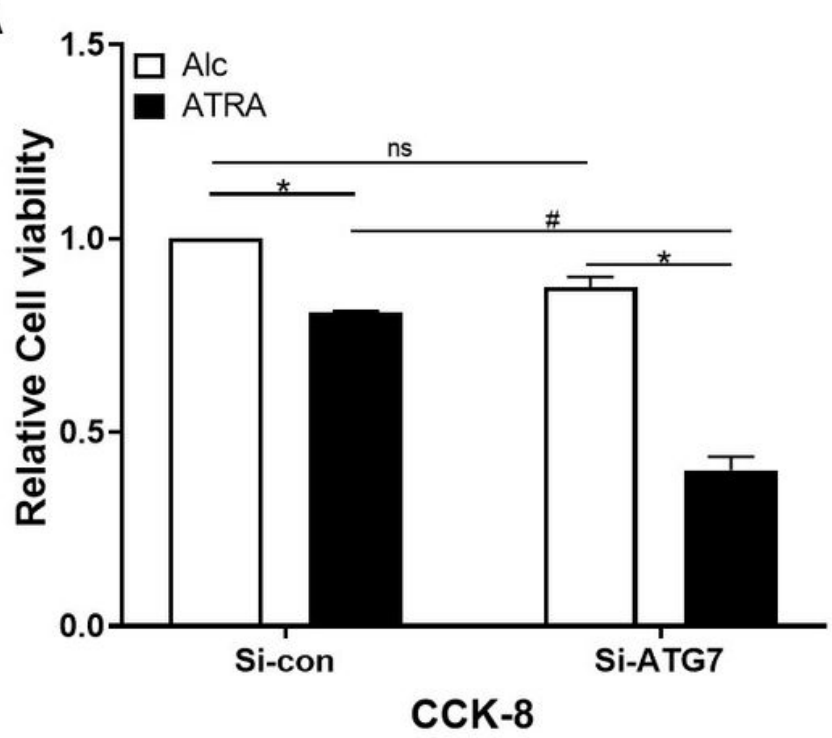

B

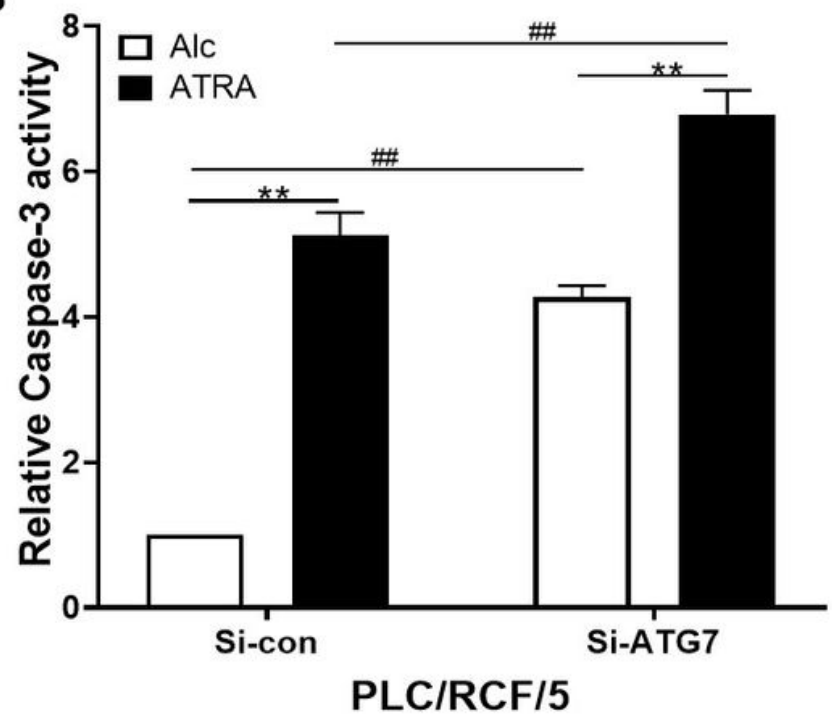

$A^{\prime}$

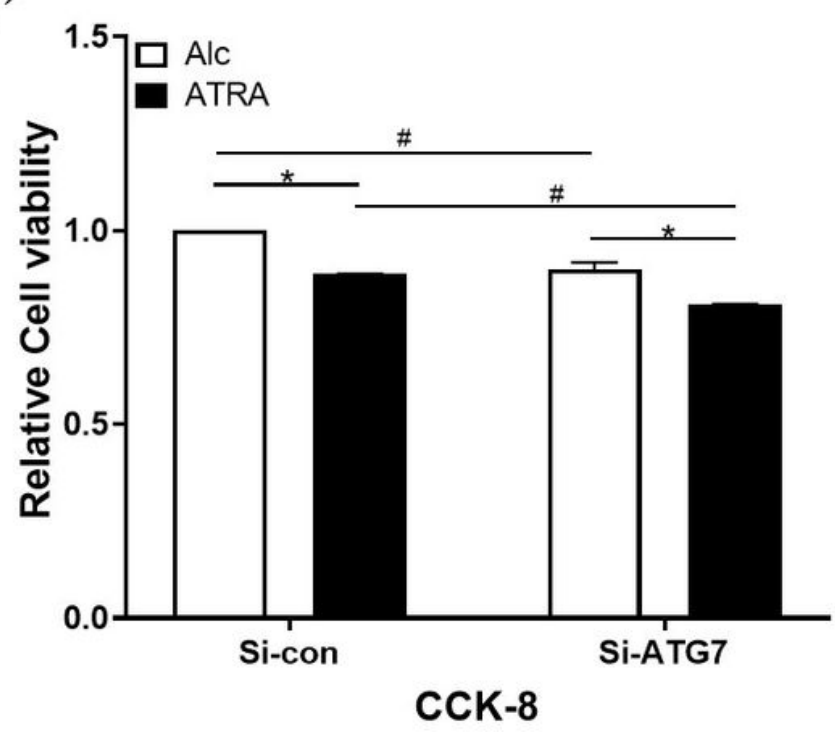

B'

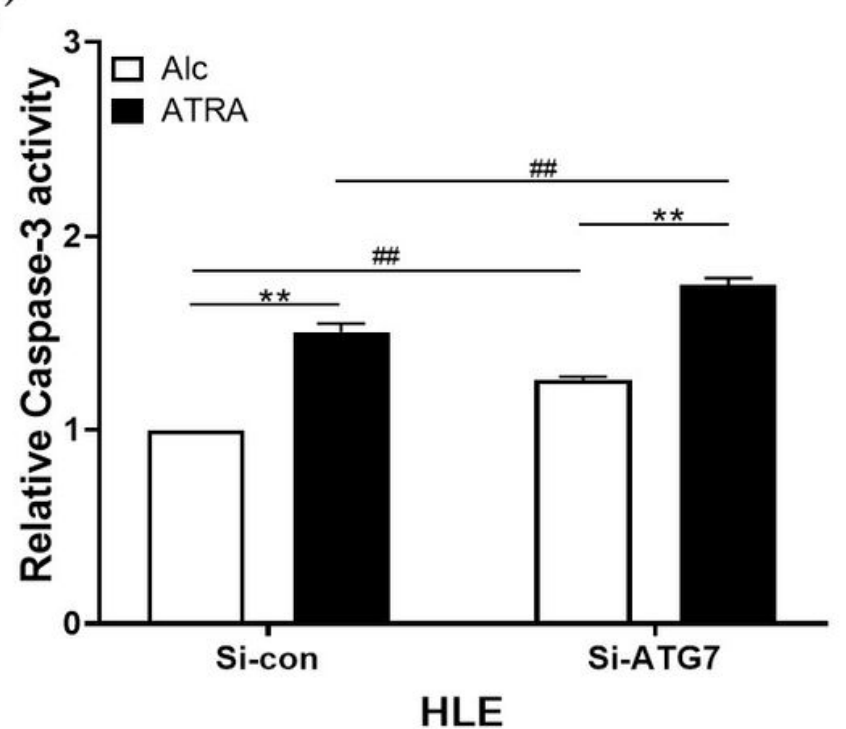

Figure 5

ATG7 prevented ATRA-induced HCC cell apoptosis. PLC/PRF/5 and HLE cells transfected with siRNAs (sicon and si-ATG7) for $24 \mathrm{~h}$, then remove the supernant and added ATRA at final concertaion at 40uM treated for $24 \mathrm{~h}$. PLC/PRF/ 5 cells $(A)$ and HLE (A') cell viability was determinated by CCK-8 assays. $\mathrm{PLC} / \mathrm{PRF} / 5$ cells $(\mathrm{B})$ and HLE (B') cell apoptosis were calculated with caspase-3 activity. 
A
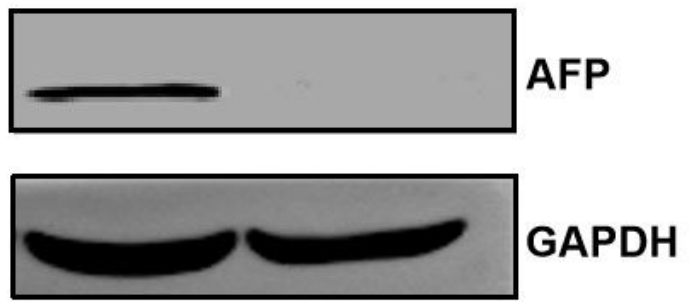

PLC/RCF/5 HLE
B
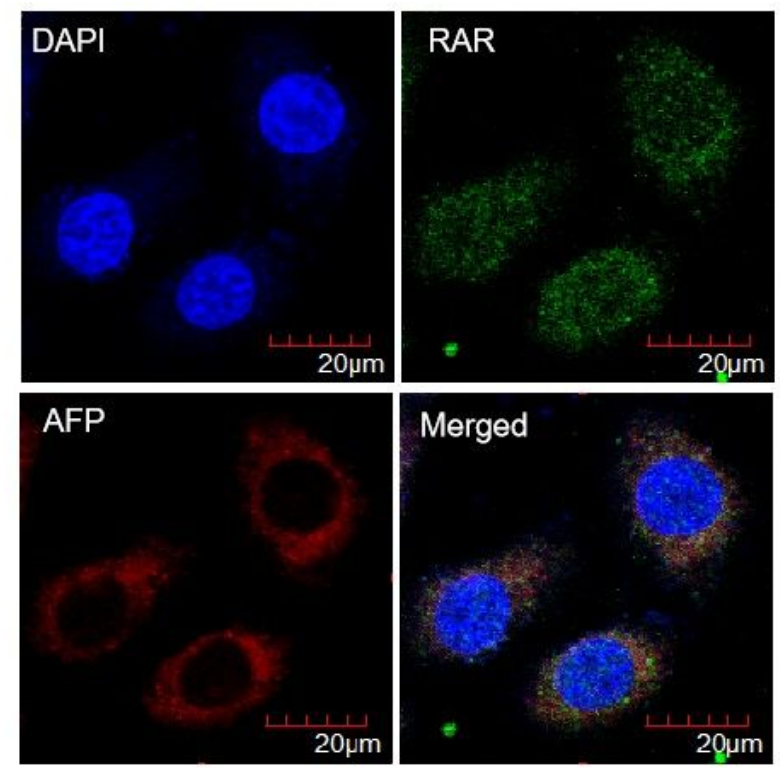

IP

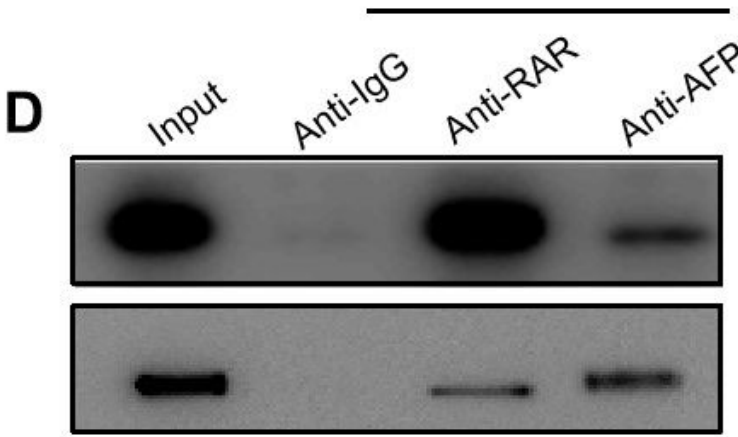

AFP

RAR
C
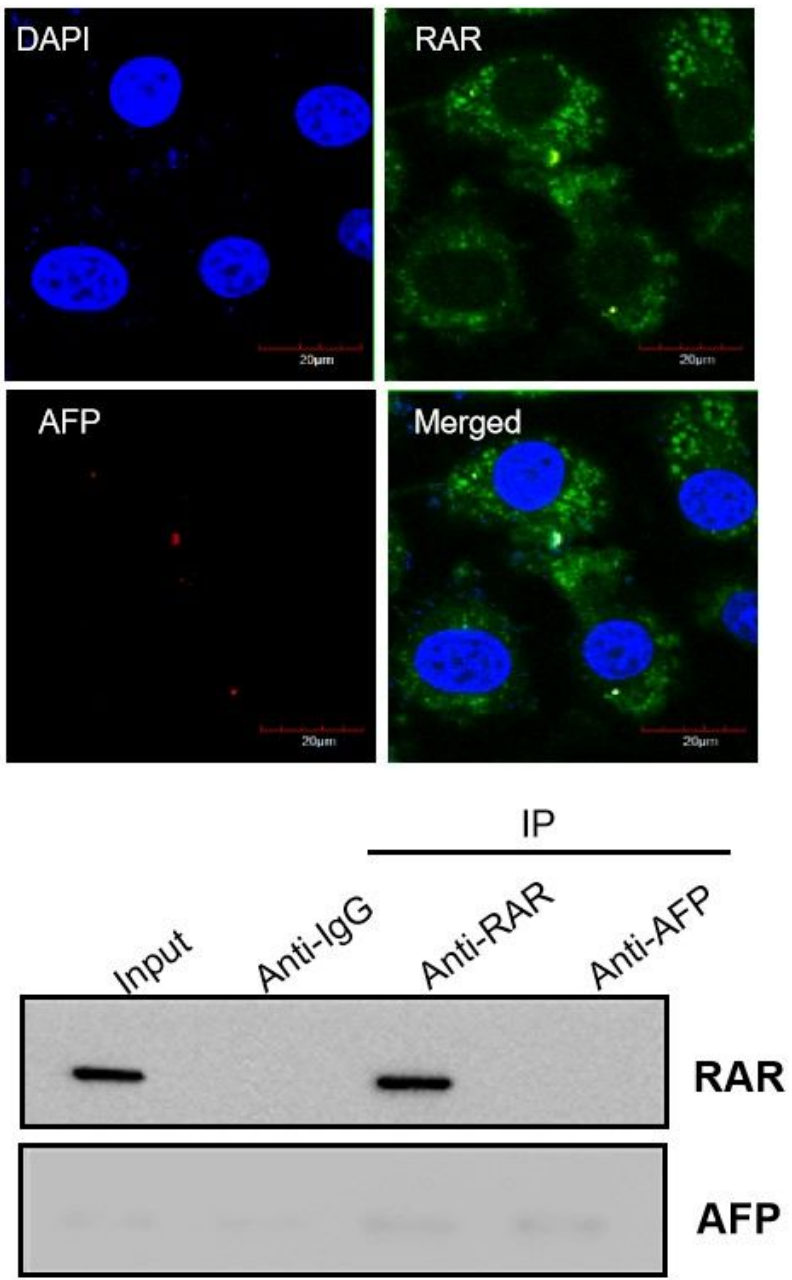

HLE

\section{PLC/RCF/5}

\section{Figure 7}

AFP interacted with RAR in cytoplasm of HCC cells. A. Western blotting was used for analysis of expression of AFP in PLC/PRF/5 and HLE cells. Expression and localization of AFP and RAR were analyzed with confocal microscopy in PLC/PRF/5 (B) and HLE (C) cells. In PLC/PRF/5 (D) and HLE (E) cells, Co-IP was employed to detect the interaction of AFP and RAR. The images captured by confocal microscope are representatives of experiments that were repeated at least three times. 
A
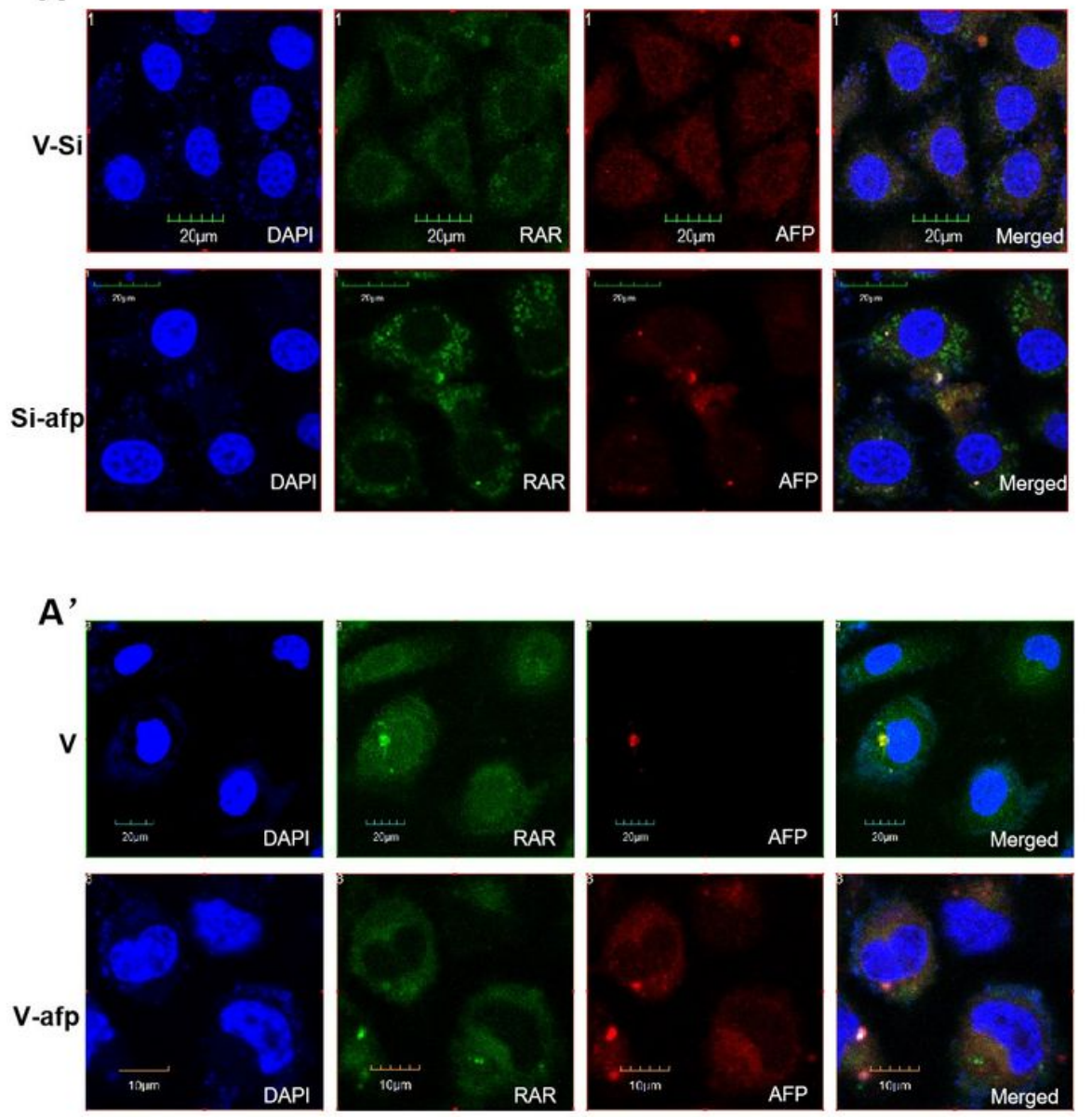
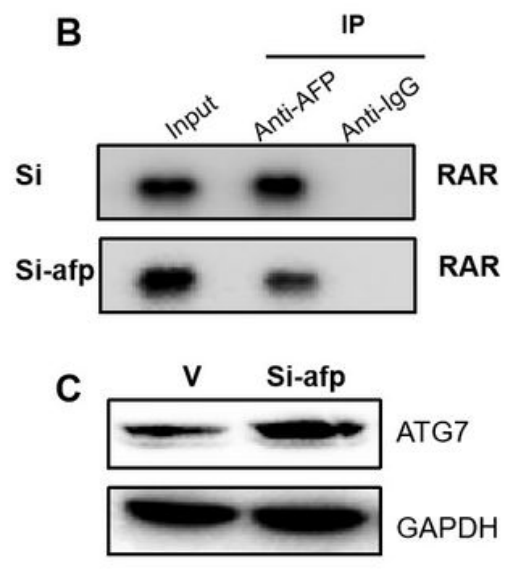

$\mathrm{PLC} / \mathrm{RCF} / 5$

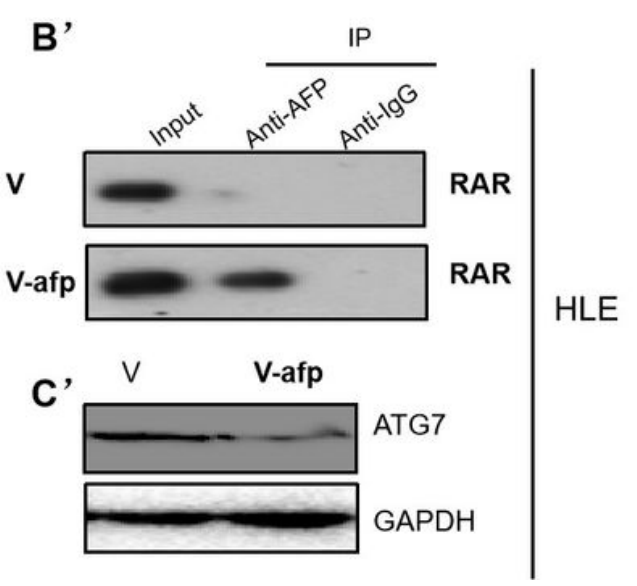

\section{Figure 9}

AFP attenuated RAR mediated of ATG7 expression in HCC cells. Localization of AFP (red) and RAR (green) in AFP-shRNA923-transfected PLC/PRF/5 cells (A) and pcDNA3.1-afp-transfected HLE cells (A') were detected with immunofluorescence and observed with confocal microscopy. Co-IP was carried out to detect the interaction between AFP and RAR in AFP knockdown or ectopic expression in PLC/PRF/ 5 cells (B) or HLE cells ( $\left.B^{\prime}\right)$, respectively. Effects of AFP knockdown or ectopic expression on ATG7 expression in $\mathrm{PLC} / \mathrm{PRF} / 5$ cells $(\mathrm{C})$ and HLE (C') cells were analyzed by Western blotting.

\section{Supplementary Files}

This is a list of supplementary files associated with this preprint. Click to download.

- S2.jpg

- S1.jpg

- S1.jpg

- S2.jpg 